\title{
La disposición clasista de los entornos de residencia en las principales conurbaciones de México. Una aproximación desde las categorías de ocupación laboral
}

\author{
Fernando Calonge Reillo \\ Universidad de Guadalajara, México \\ fercalonge@yahoo.es
}

Recepción: 13-03-2019

Aceptación: 29-04-2020

Publicación: 09-06-2020

\section{Resumen}

En los últimos decenios, se ha incrementado el interés por recuperar los análisis en términos de clase social vinculados a la posición de los diferentes grupos en los espacios urbanos. Dentro de esta tendencia, cobran especial fuerza las investigaciones que se focalizan en analizar la constitución de las clases sociales en los entornos de residencia. El objetivo de este artículo consiste en explorar de qué forma se produce la distribución clasista de la metrópoli atendiendo especialmente a los entornos de residencia en las principales conurbaciones mexicanas. Para hacerlo, se realiza una explotación y un análisis de la Encuesta Nacional de Ingresos y Gastos de los Hogares 2016 (ENIGH), México, tomando como variable independiente y expresiva de la clase social las agrupaciones de los hogares según la ocupación de la jefatura de hogar, y como variables dependientes, distintos indicadores sobre la ubicación de las viviendas, la habitabilidad o las formas de acceso a las residencias. El artículo sitúa la discusión sobre las clases sociales y el entorno de residencia en el contexto latinoamericano, y su principal contribución estriba en resaltar la existencia de una escalonada y bien definida estructura clasista en el disfrute del espacio urbano, con los hogares encabezados por los directivos y, en menor medida, los profesionales como los máximos beneficiarios. Asimismo, se destaca la existencia de importantes núcleos rurales que se están incorporando a la urbe y que, al ser mayoritariamente precarios y deficitarios, son sede para los hogares de agricultores y peones.

Palabras clave: territorios urbanos; clases sociales; segregación urbana; habitabilidad; entornos residenciales 
Abstract. Social class and housing environments in Mexico's main metropolitan areas: An approach based on occupational categories

In the last decades there has been a growing interest to recover reflections on social classes that are now dependent on the production of urban space. Within this trend, there are many contributions that analyze how social classes coalesce in residential environments. The purpose of this paper consists in exploring the class nature of the metropolis, paying special attention to the housing environments in the main Mexican conurbations. The paper is based on an analysis of the National Survey on Household Revenues and Expenditures in Mexico. We consider the independent variable that captures social class to be the head of households' occupation, and as dependent variables some indexes about housing location, living conditions or access to residences. The article reveals a well demarcated social structuring of urban space, with households headed by managers and professionals at the top, and peasants and day laborers at the rear. It also reveals that households headed by peasants and day laborers are mostly accommodated in run-down and semi-rural areas that are becoming integrated in the recent urban expansion.

Keywords: urban territories; social classes; urban segregation; living conditions; housing environments
Sumario
1. Introducción
4. Resultados
2. En enfoque de la clase social y el
5. Conclusiones
entorno residencial
Referencias bibliográficas
3. Metodología

\section{Introducción}

En los dos últimos decenios, se ha recuperado el debate sobre la existencia de las clases sociales, pero se ha ubicado en un nuevo contexto. Desde su fundación, a mediados del siglo xix, el centro de atención residía en saber cómo la esfera de la producción generaba la división clasista de la sociedad. La relación de los sujetos con los medios de producción era lo que determinaba su posición en el sistema de clases sociales. Sin embargo, en los últimos tiempos se ha buscado en las formas de habitar y vivir la ciudad un nuevo espacio para la estructuración de la sociedad. En el entorno anglosajón, el debate se ha centrado en entender cómo las clases medias han conseguido generar un sentido de clase social en la medida en que aprovechaban los recientes procesos de gentrificación urbana vividos por las principales metrópolis. Correlativamente, han aparecido estudios que se preguntaban de qué manera las clases bajas y trabajadoras construían su sentido de clase social a través de la defensa e identificación con los tradicionales barrios obreros.

En este artículo retomamos ese tipo de discusiones y las ubicamos en el espacio más próximo del entorno residencial. En particular, nos interrogamos sobre cómo las diferentes clases sociales acceden y construyen sus entornos 
residenciales, de forma que la desigualdad social acaba adquiriendo también un correlato territorial sustantivo. Nuestro objetivo es examinar de qué modo la división clasista de la sociedad se observa también en la división clasista de los entornos residenciales. Como indicador de la clase social, se ha recurrido a las categorías de ocupación laboral. La investigación se sustenta en la explotación y el análisis estadístico de la Encuesta Nacional de Ingresos y Gastos de los Hogares (ENIGH) 2016, que realiza el Instituto Nacional de Estadística y Geografía (INEGI) de México, para el caso de las principales conurbaciones del país: Área Metropolitana del Valle de México, de Guadalajara, de Monterrey y de Puebla-Tlaxcala. La principal aportación de nuestra investigación consiste en el descubrimiento de una bien definida y escalonada jerarquía en la ocupación de los entornos de residencia, y en el descubrimiento de que las posiciones inferiores ocupan los entornos habitacionales semirrurales que recientemente se incorporan al crecimiento de las principales metrópolis en el contexto latinoamericano. De esta forma, a las polaridades clasistas que habitualmente se presentan en el espectro urbano, habría que añadir otras que abarcan también los contextos semirrurales en proceso de conurbación y que acentúan las diferencias sociales y espaciales vividas.

\section{En enfoque de la clase social y el entorno residencial}

La relación entre la estructuración de clases y la disposición del territorio urbano ocupó el centro de atención desde la fundación de la sociología urbana. La incorporación del enfoque ecológico en el entorno urbano que realizó la Escuela de Chicago motivó que autores como McKenzie (1924: 297) o Park (2015: 579) señalaran que las clases más adineradas ocupaban los espacios de mayor valor de la urbe y dejaban a las clases pobres los espacios más desvalorizados. En su definición más clásica (Burgess, 1925: 51), esta ocupación comportaba una estructura concéntrica del territorio y de la distribución de las clases sociales, determinada por la variación de los valores ecológicos según nos alejamos del centro. Dentro de esta misma escuela, otros autores señalaron que aspectos como las diferencias culturales también eran preservados por la estructura del espacio urbano junto con los meramente económicos (Wirth, 1928: 283).

Un tiempo después, los teóricos urbanos de inspiración marxista abandonaron ese esquema interpretativo basado en la competencia económica y lo sustituyeron por otro asentado en la explotación económica. Para ellos, la ciudad era observada como el arreglo que aseguraba la reproducción de la mano de obra indispensable para mantener unas relaciones de producción basadas en la explotación (Castells, 1977). O bien la contemplaban como el escenario de la acumulación capitalista que permitía sortear las crisis recurrentes en los circuitos primarios de producción de mercancías (Harvey, 1985) a través de la inversión inmobiliaria, que generaba graves procesos de desarrollo desigual. En esta tradición, las particularidades de los procesos urbanos quedaban subsumidas bajo lógicas más generales de acumulación capitalista. 
Sin embargo, en las dos últimas décadas han aparecido una serie de contribuciones que analizan la estructuración clasista de la ciudad por fuera de los ciclos de acumulación y producción económicos, y se centran en analizar por qué compartir un espacio es clave para el proceso de objetivación de las clases sociales (Watt, 2006: 779; Miller et al., 1998: 137). El hecho de que sujetos similares compartan un mismo territorio ayuda a distinguirse como clase social y a alcanzar conciencia de su particularidad (Avalle, 2009: 6; Rodenstedt, 2014: 42).

$\mathrm{Al}$ analizar la conformación clasista en el espacio urbano, lo primero que resalta es una gran desigualdad de entornos residenciales: cruzar una calle puede suponer cambiar radicalmente de realidades espaciales y sociales y adentrarse en un contexto clasista opuesto (Rodenstedt, 2014: 137). El tipo y la calidad de las viviendas contrastan agudamente (Lejeune et al., 2016: 505), aunque se dispongan entre secciones contiguas de la ciudad (Meng y Hall, 2006: 432).

En un contexto donde la volatilidad laboral dificulta la formación de la clase social, se ha señalado que las clases medias construyen su unidad a través de la creación de estilos de vida compartidos, en las formas particulares de consumir el espacio urbano (Urry, 1995: 209; Allen, 2008: 52; Fleischer, 2007: 288). Solo estas clases medias y altas tienen mayores posibilidades de elección del espacio de residencia. Para estos grupos sociales, se ha indicado que la elección del lugar de residencia implica posicionarse dentro de un campo de diferencias sociales que incide en el sentido de clase que se alcanzará (Benson, 2014: 3.098; Butler y Watt, 2007: 86; Hunter, 1987: 217). De este modo, las clases medias y altas imponen en todo el entorno urbano un canon sobre modelos de hábitat y de consumo que sirve para clasificar al resto de las posiciones sociales, que tienen mucho más constreñida su capacidad de elección (Gato, 2014: 64).

El proceso tiene una dimensión cognitiva y una dimensión moral. Desde la cognitiva, las clases altas ganarían un sentido del sí desde la selección simbólica de sus estilos de vida (Savage, 2013: 157; Davidson, 2007: 502), a través del consumo de entornos residenciales estilizados con alto valor estético (Pow, 2009: 375), mientras que las clases bajas lo harían desde una condición más manifiesta de arrojamiento espacial (Savage, 2010: 132), que les permitiría extraer una memoria compartida (Blokland, 2001: 271; Lager et al., 2013: 57; Paton, 2014: 121) de haber convivido con sujetos similares durante un largo tiempo (Matthews, 2015: 35).

La dimensión moral implica que los sujetos no solo se diferencien de otras clases sociales por compartir y percibir un lugar diferenciado, sino por extraer de él los lazos emocionales y morales que los consolidan como unidad (Dowling, 2009: 834). En el posesionamiento de un lugar, los integrantes de la clase social aprenden a reclamarlo como propio y a extraer de él ciertas formas de vida y de comportamiento particulares (Savage, 2010: 115). Este posesionamiento sustantivo del espacio depara que en todo el contexto urbano aparezcan identificaciones clasistas de espacios de respetabilidad y de indecencia (Rhodes, 2012: 698). 
$\mathrm{Al}$ analizar las metrópolis presentes, se ha insistido en que esa diferenciación no se produce solo entre distritos o polígonos de la ciudad claramente demarcados; al contrario, se señala que una aguda fragmentación permea todos los distritos de la ciudad, porque no es posible identificar grupos clasistas homogéneos (Davidson, 2007: 501), porque grupos similares se disponen dispersos y atomizados a lo largo de la ciudad (Selzer y Heller, 2010: 186) y porque incluso dentro de un vecindario se advierten esfuerzos denodados por establecer fronteras y divisiones de clase (Pinkster, 2014: 813). Esta situación tan volátil en la identificación socioespacial de la realidad clasista del grupo social motiva que los sujetos se esfuercen por sostener su propia afiliación remarcando y ampliando la distancia respecto a las demás clases sociales (Allen, 2008: 46).

Como se puede constatar en esta estructuración clasista de la ciudad, las fórmulas son múltiples. Así, las clases altas suelen decantarse por entornos de alta calidad ambiental, bien conectados territorialmente, con bajos niveles de contaminación y bajas densidades, que favorecen un sentido de mayor privacidad (Fleischer, 2007: 292). El escaso poder adquisitivo de las clases bajas condiciona que tengan que ubicarse en espacios menos centrales (Ward, 2004: 781) y, en los países en desarrollo, vivan en lugares mayoritariamente periféricos (Libertun de Duren, 2018: 411). Dado que es habitual que la ocupación del territorio de estas clases se dé bajo la fórmula de la irregularidad (Gilbert y Ward, 1985: 56), es frecuente que dispongan de un acceso solo parcial a los servicios urbanos más básicos (Shefner, 2000: 346), lo que ofrece un fuerte contraste con los otros espacios de la metrópoli ocupados por las rentas más elevadas.

Otra de las fórmulas que imponen una diferencia clasista en la apropiación del entorno de residencia es la forma de acceso a la vivienda, sobre todo en un contexto en que se evidencia una mayor importancia del mercado como forma para comprar una la residencia (McKee y Muir, 2013: 2). En esta situación en la que se observa un incremento generalizado del precio de la vivienda (Wood y Ong, 2011: 1.105), la tenencia bajo régimen de propiedad ofrece una ventaja relativa muy importante (McIntyre y McKee, 2012: 237), sobre todo para las clases altas y medias. Por el contrario, las rentas más bajas son las que tienen más dificultad para afrontar los crecientes precios (Ben-Shadar et al., 2018; Lens, 2018: 1.616; Sunega y Lux, 2016: 703), lo que incide en que tengan más dificultades para poder acceder como propietarios a las viviendas (Conley, 2001: 272).

En general, hay acuerdo en la literatura sobre la normalización de la figura de propietario como fórmula prioritaria de acceso a la vivienda (Kelafas, 2003: 98), y a la hora de señalar que los propietarios, sobre todo de clases medias y altas, tienen mejores condiciones de bienestar (Filandri y Olagnero, 2014: 988). Además, el hecho de que los sujetos de clases bajas tengan más dificultades para hacerse propietarios de viviendas lastra sus posibilidades de ascenso social, debido a que deben emplear una parte importante de sus presupuestos en los alquileres y rentas, lo que compromete el resto de gastos en educación, transporte, etcétera (Bunting et al., 2004: 379). 
Además, en las ciudades contemporáneas se puede apreciar una clara polarización respecto al equipamiento y a las características de las residencias (Aliu y Ajala, 2015: 281). En términos de espacio, las clases más altas suelen incluir entre uno de sus criterios de compra disfrutar de viviendas más amplias y cómodas (Bridge, 2003: 2.552; Bacqué et al., 2014: 1.220), mientras que las clases bajas tienen que conformarse con viviendas pequeñas (Lejeune et al., 2016: 502), donde la situación de hacinamiento suele ser más grave (Pla et al., 2018: 210). De igual manera, se ha puesto de manifiesto que las viviendas de las clases bajas cuentan con menos baños, lo que dificulta sus condiciones de higiene (Lejeune et al., 2016: 503). También se ha destacado que la posesión de determinados aparatos domésticos incide en el disfrute de calidades de la vivienda muy distintas (Meng y Hall, 2006: 419), o que los consumos de energía son muy diferentes según las clases sociales (McLoughlin et al., 2012: 245), algo que, en los extremos inferiores de la estructura social, suele expresarse en situaciones de pobreza energética (Aristondo y Onaindia, 2018: 432).

La investigación sobre la disposición clasista de los entornos residenciales tiene lugar en un contexto urbano latinoamericano donde la iniciativa privada ha tomado el protagonismo en la planificación territorial. Así pues, las desarrolladoras inmobiliarias suelen ubicar sus nuevas promociones de vivienda en las periferias metropolitanas, al encontrar suelo más barato y poder generar economías de escala más fácilmente (Libertum de Duren, 2018: 416), lo que ha ocasionado una amplia expansión del territorio urbanizado en las tres últimas décadas. Buena parte de la diferenciación clasista en el territorio se ubica en espacios limítrofes y semirrurales. Estos espacios están recibiendo una atención creciente, bien desde el punto de vista del planeamiento y el territorio (RojasCaldelas et al., 2010; Aguilar y Ward, 2003), del tejido y las relaciones sociales (Méndez-Lemus y Vieyra, 2017) o de las movilidades cotidianas (Oviedo Hernández y Titheridge, 2015).

Desde el punto de vista de la clase social, esta reciente urbanización de la periferia ha acarreado dos tipos muy diferenciados de hábitats. Por un lado, tenemos el ejemplo de espacios que por sus condiciones de calidad ambiental y paisajística han servido para la concentración de enclaves de clases medias y altas (Nates et al., 2019: 148). La dominante, sin embargo, es el establecimiento de residencias para poblaciones de bajos recursos que se ubican en ocasiones en zonas de riesgo ambiental y escasamente dotadas de servicios e infraestructura (Cardoso, 2018), lo que compromete seriamente sus condiciones de habitabilidad (Zulaica y Oriolani, 2018: 669).

Este artículo contribuye a aportar conocimiento sobre la escenificación de la diferencia clasista en los territorios urbanos en el contexto latinoamericano, donde este tipo de investigaciones no es demasiado abundante. Para hacerlo, retomaremos las ya mencionadas variables sobre los entornos residenciales, para ver de qué manera expresan esa naturaleza clasista en la división del espacio, y lo haremos en el contexto de las grandes metrópolis mexicanas, caracterizadas por su reciente expansión hacia los bordes semirrurales. 


\section{Metodología}

Los resultados del presente trabajo se basan en una explotación y un análisis estadístico de la Encuesta Nacional de Ingresos y Gastos de los Hogares 2016 (ENIGH 2016), que realiza el Instituto Nacional de Estadística y Geografía, México (INEGI). La encuesta tiene cobertura en todo el territorio nacional, pero para rescatar el análisis en términos urbanos se procedió a incluir solo los casos de las cuatro principales conurbaciones del país: Área Metropolitana del Valle de México, de Guadalajara, de Monterrey y de Puebla-Tlaxcala. La composición de las distintas áreas metropolitanas se realizó agregando los municipios que guardan continuidad urbana en cada una de las metrópolis señaladas, de acuerdo con la delimitación de las zonas metropolitanas que realiza el INEGI (2018). ${ }^{1}$

En nuestro intento por realizar una aproximación al análisis en términos de clase social, hemos recurrido a la variable ocupación siguiendo la recomendación que en su momento hicieran Solís y Boado (2016). Por tanto, el uso de otras variables o combinación de variables podría arrojar resultados divergentes a los nuestros. La operacionalización de las variables utilizadas en el análisis puede visualizarse en la tabla 1.

Tabla 1. Cuadro resumen de las variables empleadas en el análisis

\begin{tabular}{|c|c|c|}
\hline Variables & Tipo & Valores \\
\hline Ocupación & Independiente & $\begin{array}{l}\text { Agricultores, peones, manuales cualificados } \\
\text { y operarios de maquinaria, comerciantes y } \\
\text { no cualificados de servicios personales, } \\
\text { auxiliares administrativos, profesionales } \\
\text { cualificados y directivos. }\end{array}$ \\
\hline Tenencia de la vivienda & Dependiente & Alquilada, prestada, propia \\
\hline Financiamiento de la vivienda & Dependiente & $\begin{array}{l}\text { Créditos de instituciones públicas, banca } \\
\text { privada, con recursos propios }\end{array}$ \\
\hline $\begin{array}{l}\text { Compra vivienda hecha o } \\
\text { mandada construir }\end{array}$ & Dependiente & $\begin{array}{l}\text { La compra hecha, la manda construir, } \\
\text { autoconstrucción, otra manera. }\end{array}$ \\
\hline Tamaño de la localidad & Dependiente & $\begin{array}{l}\text { Menos de } 2.500 \text {, de } 2.500 \text { a } 99.999 \text {, } \\
100.000 \text { y más }\end{array}$ \\
\hline Número de residentes por cuarto & Dependiente & Numérica \\
\hline Número de baños completos & Dependiente & Numérica \\
\hline Renta trimestral de la vivienda & Dependiente & Numérica \\
\hline $\begin{array}{l}\text { Peso del gasto de la vivienda } \\
\text { en total de ingresos }\end{array}$ & Dependiente & Numérica \\
\hline $\begin{array}{l}\text { Gastos trimestrales en energía } \\
\text { de la vivienda }\end{array}$ & Dependiente & Numérica \\
\hline
\end{tabular}

Fuente: elaboración propia.

1. Encuesta presencial en viviendas, en el ámbito geográfico de todo el territorio nacional mexicano. Muestreo aleatorio estratificado por tamaños de población, que integra un total de 81.515 viviendas. Afijación proporcional de los elementos muestrales a los estratos. Margen de error de $\pm 0,4 \%$, con un margen de confianza del $95 \%$ en las estimaciones del agregado a nivel nacional. La submuestra generada para la presente investigación, que integra las principales metrópolis del país, está compuesta por un total de 5.616 elementos muestrales. 
Se utilizaron diversos estadísticos para calibrar las asociaciones y relaciones existentes entre la variable independiente y las dependientes. Para variables de naturaleza nominal, se utilizó el estadístico de asociación $\mathrm{V}$ de Cramer, que varía de 0 a 1 en función de la intensidad de la asociación, y el estadístico de correlación Rho de Spearman cuando las variables son de naturaleza ordinal. Asimismo, se utilizaron regresiones logísticas para medir de qué forma la variable independiente de las ocupaciones incidía en la probabilidad de que la vivienda asumiera determinadas características particulares. Cuando estábamos ante unidades cuantitativas de medida, se utilizaron modelos de análisis de la varianza (ANOVA) para estudiar si las diferencias de medias eran significativas para cada uno de los siete grupos de ocupaciones. En la presentación de los resultados se indicará qué estadístico ha sido utilizado en cada caso concreto.

\section{Resultados}

\subsection{Ocupación y acceso a la vivienda}

En principio, se aprecia una leve asociación entre las categorías de ocupación y el tipo de tenencia de la vivienda $(\mathrm{V}$ de Cramer $=0,155)$. Si atendemos a la distribución de los porcentajes que se muestran en la tabla 1, podemos extraer algunas conclusiones.

De forma que contrasta con la revisión de la literatura, encontramos que hay un mayor nivel de propietarios entre los hogares con jefatura de agricultores y de peones que en los hogares encabezados por directivos, profesionales cualificados y auxiliares administrativos. En estas tres categorías ocupacionales, hay una sobrerrepresentación de los hogares que tienen casa propia pero todavía la están pagando. El acceso a la propiedad, frente a lo documentado en otros contextos, no sería una marca de clase social.

Esta particularidad se pone mejor en contexto cuando observamos los datos de las tablas 3 y 4 .

Tabla 2. Distribución porcentual de los hogares de la tenencia de su vivienda, según grupos de ocupaciones

\begin{tabular}{lrrrr}
\hline & \multicolumn{4}{c}{ Propia } \\
Ocupaciones & Alquilada & Prestada & pagándose & Propia \\
\hline Agricultores & $1,9 \%$ & $12,6 \%$ & $1,1 \%$ & $84,3 \%$ \\
Peones & $15,8 \%$ & $21,1 \%$ & $5,2 \%$ & $57,9 \%$ \\
Manuales cualificados y operarios de maquinaria & $16,3 \%$ & $15,0 \%$ & $15,6 \%$ & $53,1 \%$ \\
Comerciantes y no cualificados servicios personales & $21,0 \%$ & $16,2 \%$ & $14,3 \%$ & $48,6 \%$ \\
Auxiliares administrativos & $22,9 \%$ & $14,5 \%$ & $25,1 \%$ & $37,4 \%$ \\
Profesionales cualificados & $22,1 \%$ & $11,7 \%$ & $21,0 \%$ & $45,3 \%$ \\
Directivos & $25,7 \%$ & $7,9 \%$ & $22,0 \%$ & $44,5 \%$ \\
Total & $17,7 \%$ & $15,6 \%$ & $13,6 \%$ & $53,1 \%$ \\
\hline
\end{tabular}

Fuente: elaboración propia a partir de la Encuesta Nacional de Ingresos y Gastos en los Hogares (ENIGH 2016), Instituto Nacional de Estadística y Geografía (INEGI). 
Tabla 3. Distribución porcentual de los hogares del proceso de construcción según grupos de ocupación

\begin{tabular}{lcc}
\hline Ocupaciones & Se compra hecha & $\begin{array}{c}\text { La construye o } \\
\text { manda construir }\end{array}$ \\
\hline Agricultores & $6,7 \%$ & $93,3 \%$ \\
Peones & $22,6 \%$ & $77,4 \%$ \\
Manuales cualificados y operarios de maquinaria & $42,2 \%$ & $57,8 \%$ \\
Comerciantes y no cualificados servicios personales & $46,2 \%$ & $53,8 \%$ \\
Auxiliares administrativos & $67,9 \%$ & $32,1 \%$ \\
Profesionales cualificados & $63,4 \%$ & $36,6 \%$ \\
Directivos & $70,7 \%$ & $29,3 \%$ \\
Total & $44,9 \%$ & $55,1 \%$ \\
\hline
\end{tabular}

Fuente: elaboración propia a partir de la ENIGH 2016 (INEGI).

Tabla 4. Distribución porcentual de los hogares del tipo de financiamiento en la adquisición de la vivienda, según ocupaciones.

\begin{tabular}{lrc}
\hline Ocupaciones & Hipoteca & Recursos propios \\
\hline Agricultores & $8,1 \%$ & $91,9 \%$ \\
Peones & $23,8 \%$ & $76,2 \%$ \\
Manuales cualificados y operarios de maquinaria & $41,6 \%$ & $58,4 \%$ \\
Comerciantes y no cualificados servicios personales & $61,9 \%$ & $38,1 \%$ \\
Auxiliares administrativos & $55,7 \%$ & $44,3 \%$ \\
Profesionales cualificados & $62,0 \%$ & $38,0 \%$ \\
Directivos & $40,5 \%$ & $59,5 \%$ \\
Total & $39,0 \%$ & $61,0 \%$ \\
\hline
\end{tabular}

Fuente: explotación propia basada en la ENIGH 2016 (INEGI).

El grado de asociación entre los grupos de ocupaciones y el proceso de construcción es más destacado que en la relación anterior $(\mathrm{V}$ de Cramer $=$ $0,333)$. Se puede comprobar que los hogares encabezados por agricultores y peones, que eran quienes tenían unos mayores niveles de propiedad, lo consiguen gracias a que mayoritariamente construyen sus propias viviendas en lugar de acudir al mercado para adquirirlas ya hechas. En el extremo opuesto, los directivos, los profesionales cualificados y los auxiliares administrativos recurren por encima de la media al mercado para comprar sus casas. Esta evidencia se ratifica cuando contemplamos las formas de financiamiento.

$\mathrm{La}$ asociación de las dos variables anteriores es apreciable (V de Cramer $=0,298)$, y añade que gracias a los ahorros y recursos propios los hogares encabezados por agricultores y peones tienen en propiedad sus casas y las han construido ellos mismos. Por el contrario, los profesionales cualificados, los auxiliares administrativos y, en este caso también, los comerciantes y trabajadores no cualificados de servicios personales parecen recurrir más a las instituciones financieras establecidas para adquirir a través del crédito hipotecario 
sus residencias. En este aspecto, los directivos, en principio por su mayor nivel adquisitivo, no recurren de una forma tan destacada a este tipo de créditos y aprovechan en buena proporción sus recursos propios para adquirir la vivienda.

Los modelos de regresión logística aplicados entre las anteriores variables son débiles desde el punto de vista explicativo. La variable independiente ocupación de la jefatura de hogar apenas consigue explicar un 8,7 \% de la varianza de la variable dependiente tenencia de la vivienda, e incurre en gruesos errores a la hora de clasificar los distintos casos, por lo que hemos desechado este modelo. Algo más ajustados son los modelos que incluyen como variables dependientes el proceso de construcción y el tipo de financiamiento. En el primer caso se consigue explicar un $15 \%$ de la variable dependiente y en el segundo un $12 \%$. Las probabilidades obtenidas se muestran en la tabla 5.

Como se observa, todos los coeficientes son significativos. Las regresiones logísticas binarias confirman algunas de las apreciaciones ya extraídas de la observación de las tablas cruzadas. Así, se destaca que los agricultores tienen un $91,7 \%$ de probabilidades menos de comprar hecha la vivienda y un $88,6 \%$ de probabilidades menos de recurrir a una hipoteca que la categoría de referencia de los comerciantes y trabajadores no cualificados de servicios personales. Los peones también presentan probabilidades negativas en esos dos aspectos respecto a esa categoría de referencia. En el extremo opuesto de la estructura ocupacional, comprobamos que los directivos tienen un $180,8 \%$ más de probabilidades de comprar hecha la vivienda y un 122,1\% más de probabilidades de recurrir a un crédito hipotecario que la categoría de referencia de los comerciantes y trabajadores no cualificados de servicios personales. Por otro lado, los auxiliares administrativos tienen un $146,9 \%$ más de probabilidades de comprar hecha la vivienda y un $312,2 \%$ más de probabilidades de financiarla a través de una hipoteca que la mencionada categoría de referencia.

Tabla 5. Opciones de comprar hecha la vivienda y de recurrir a hipoteca según ocupaciones de la jefatura de hogar

\begin{tabular}{lcccc}
\hline & $\begin{array}{c}\text { Opciones de } \\
\text { comprar hecha } \\
\text { la vivienda }\end{array}$ & Significatividad & $\begin{array}{c}\text { Opciones } \\
\text { de recurrir } \\
\text { a hipoteca }\end{array}$ & Significatividad \\
\hline Agricultores & $-91,7 \%$ & 0,000 & $-88,6 \%$ & 0,000 \\
$\begin{array}{l}\text { Peones } \\
\text { Manuales cualificados y }\end{array}$ & $-66,1 \%$ & 0,000 & $-51,7 \%$ & 0,000 \\
$\begin{array}{l}\text { operarios de maquinaria } \\
\text { Auxiliares administrativos }\end{array}$ & $-14,8 \%$ & 0,000 & $+9,5 \%$ & 0,000 \\
$\begin{array}{l}\text { Profesionales cualificados } \\
\text { Directivos }\end{array}$ & $+146,9 \%$ & 0,000 & $+312,2 \%$ & 0,000 \\
$\begin{array}{l}\text { Categoría de referencia: } \\
\text { comerciantes y no }\end{array}$ & $+180,8 \%$ & 0,000 & $+104,7 \%$ & 0,000 \\
$\begin{array}{l}\text { cualificados de servicios } \\
\text { personales }\end{array}$ & & 0,000 & $+122,1 \%$ & 0,000 \\
\hline
\end{tabular}

Fuente: elaboración propia a partir de la ENIGH 2016 (INEGI). 


\subsection{Categoría de ocupación y características fisicas de la vivienda}

$\mathrm{Al}$ analizar la distribución de los integrantes de las diferentes categorías ocupacionales por los territorios metropolitanos, advertimos la persistencia de un muy destacado porcentaje de agricultores en localidades de menos de 2.500 habitantes. También es apreciable el porcentaje de peones que viven en este tipo de localidades que denotan estar en un proceso de transición rural-urbano.

El nivel de correlación entre ambas variables medido a través del estadístico Rho de Spearman es apreciable $(0,315)$. Se constata que en las localidades conurbadas de naturaleza rural de menos de 2.500 habitantes están sobrerrepresentados los peones y, sobre todo, los agricultores. Por su parte, los directivos y los profesionales cualificados están sobrerrepresentados en las localidades eminentemente urbanas y centrales de 100.000 y más habitantes. El modelo de regresión logística multinomial realizado, aunque dista mucho de explicar un porcentaje destacado de la varianza de la variable dependiente tamaño de localidad (solo explica el 12,1\%), ratifica alguna de estas apreciaciones.

Según se aprecia, las categorías de agricultores y de peones arrojan probabilidades negativas muy destacadas de vivir en poblaciones de 100.000 y más habitantes en comparación con los comerciantes y trabajadores no cualificados de servicios personales. En concreto, los agricultores tienen un 99,8 \% menos probabilidades de vivir en este tipo de hábitat que la categoría de referencia. En el escalafón opuesto, los directivos tienen un 434,2 \% más de probabilidades de vivir en este tipo de localidades eminentemente urbanas en comparación con los comerciantes y trabajadores no cualificados de servicios personales. Todos los coeficientes encontrados son estadísticamente significativos.

Aparte de residir en entornos consolidados desde el punto de vista urbano, los directivos y, en menor medida, los profesionales de las grandes conurbaciones mexicanas gozan de condiciones de habitabilidad mucho más favorables que el resto de las categorías de ocupación. Esto es así después de analizar las varianzas en la distribución de medias de residentes por cuarto y de número de baños completos de los diferentes grupos de hogares según las jefaturas.

Tabla 6. Distribución porcentual de los hogares según ocupación de la jefatura de hogar, en las distintas localidades según tamaño.

\begin{tabular}{lccc}
\hline Ocupaciones & $\begin{array}{c}\text { Menos } \\
\text { de 2.500 }\end{array}$ & $\begin{array}{c}\text { De 2.500 } \\
\text { a 99.999 }\end{array}$ & $\begin{array}{c}100.000 \\
\text { y más }\end{array}$ \\
\hline Agricultores & $52,8 \%$ & $44,7 \%$ & $2,5 \%$ \\
Peones & $10,9 \%$ & $26,7 \%$ & $62,4 \%$ \\
Manuales cualificados y operarios de maquinaria & $6,2 \%$ & $23,3 \%$ & $70,5 \%$ \\
Comerciantes y no cualificados servicios personales & $3,5 \%$ & $23,8 \%$ & $72,7 \%$ \\
Auxiliares administrativos & $4,7 \%$ & $28,3 \%$ & $67,0 \%$ \\
Profesionales cualificados & $2,5 \%$ & $18,2 \%$ & $79,3 \%$ \\
Directivos & $0,7 \%$ & $21,3 \%$ & $77,9 \%$ \\
Total & $6,9 \%$ & $24,0 \%$ & $69,1 \%$ \\
\hline
\end{tabular}

Fuente: elaboración propia a partir de la ENIGH 2016 (INEGI). 
Tabla 7. Opciones de residir en localidades de 100.000 y más habitantes según ocupaciones de la jefatura de hogar

\begin{tabular}{lcc}
\hline Ocupaciones & $\begin{array}{c}\text { Opciones de } \\
\text { comprar hecha } \\
\text { la vivienda }\end{array}$ & Significatividad \\
\hline Agricultores & $-99,8 \%$ & 0,000 \\
Peones & $-72,3 \%$ & 0,000 \\
Manuales cualificados y operarios de maquinaria & $-45,1 \%$ & 0,000 \\
Auxiliares administrativos & $-31,1 \%$ & 0,000 \\
Profesionales cualificados & $+57,4 \%$ & 0,000 \\
Directivos & $+434,2 \%$ & 0,000 \\
Categoría de referencia: comerciantes y no cualificados & & \\
de servicios personales & & \\
\hline
\end{tabular}

Fuente: elaboración propia a partir de la ENIGH 2016 (INEGI).

Tabla 8. Diferencias de medias de número residentes por cuarto y de baños completos en la vivienda y según grupos de ocupación

\begin{tabular}{lcc}
\hline Ocupaciones & $\begin{array}{c}\text { Diferencia de } \\
\text { medias número } \\
\text { de residentes por } \\
\text { cuarto }\end{array}$ & $\begin{array}{c}\text { Diferencia de } \\
\text { medias número } \\
\text { de baños } \\
\text { completos }\end{array}$ \\
\hline Agricultores & 1,33 & 0,51 \\
Peones & 1,47 & 0,70 \\
Manuales cualificados y operarios de maquinaria & 1,29 & 0,94 \\
Comerciantes y no cualificados servicios personales & 1,09 & 1,01 \\
Auxiliares administrativos & 0,96 & 1,09 \\
Profesionales cualificados & 0,90 & 1,28 \\
Directivos & 0,85 & 1,63 \\
Total & 1,8 & 1,00 \\
\hline Fil
\end{tabular}

Fuente: elaboración propia a partir de la ENIGH 2016 (INEGI).

El estadístico de Levene aplicado para analizar las varianzas de las medias de residentes por cuarto y de baños completos por vivienda según las ocupaciones de las jefaturas de hogar no ha permitido confirmar la hipótesis nula de homogeneidad de varianzas como exige la aplicación de los modelos ANOVA. Por eso se aplicó la prueba más robusta de diferencia de medias de Brown-Forsythe y se pudo confirmar la significatividad de las diferencias de medias encontradas.

Las diferencias de medias nos indican que especialmente los peones, los agricultores y los trabajadores manuales cualificados y operarios de maquinaria tienen peores condiciones de hacinamiento, pues en sus viviendas existe un promedio de $1,47,1,32$ y 1,29 residentes por cuarto. Los directivos, los profesionales cualificados y los auxiliares administrativos disponen de mucho 
más espacio al ser el índice de residentes por cuarto de 0,85, 0,9 y 0,96 respectivamente.

Cuando se observa la disponibilidad de baños, se reproduce esa escala descendente desde la posición de los directivos. Esta ocupación tiene una media de 1,63 baños completos por vivienda, los profesionales cualificados 1,28 y los auxiliares administrativos 1,09. Lejos de la media de un baño completo por hogar, y en el extremo de peores condiciones de habitabilidad, se encuentran los peones $(0,7$ baños completos por vivienda) y, sobre todo, los agricultores (0,51 baños completos por vivienda). Si recordamos la mayor presencia de estas dos categorías ocupacionales, especialmente de los agricultores, en las localidades de menos de 2.500 habitantes, podemos concluir que las condiciones de habitabilidad de tipo rural exacerban la polarización territorial en las principales conurbaciones mexicanas.

\subsection{Ocupaciones y valor de las residencias}

Una de las vías para materializar la distribución clasista por el territorio urbano suelen ser los gastos en que se incurre para hacerse con una vivienda.

La primera columna indica la diferencia de medias del valor trimestral de la renta de la vivienda para el hogar. Incluye el valor efectivo del alquiler de los hogares. En el caso de que el hogar tenga otro tipo de tenencia de vivienda distinto al alquiler, incluye el valor estimado de lo que costaría. La distribución de las varianzas no fue homogénea, por lo que se aplicó el estadístico de Brown-Forsythe para confirmar que las diferencias de medias fueran estadísticamente significativas. Los resultados son claros respecto a la posición en el mercado inmobiliario de las diferentes categorías de ocupación. Quienes se sitúan en la posición inferior son los agricultores, dado que el valor en alquiler

Tabla 9. Diferencias de medias de valor de renta de la vivienda, proporción de gasto de la vivienda sobre ingresos y proporción corregida de gasto de la vivienda sobre ingresos según grupos de ocupaciones.

\begin{tabular}{lccc}
\hline Ocupaciones & $\begin{array}{c}\text { Valor de } \\
\text { la renta } \\
\text { trimestral de } \\
\text { la vivienda }\end{array}$ & $\begin{array}{c}\text { Proporción } \\
\text { del gasto } \\
\text { en vivienda } \\
\text { sobre } \\
\text { ingresos }\end{array}$ & $\begin{array}{c}\text { Proporción } \\
\text { corregida } \\
\text { del gasto en } \\
\text { vivienda sobre } \\
\text { ingresos }\end{array}$ \\
\hline Agricultores & $3.431,32$ & 0,55 & 20,80 \\
Peones & $4.182,82$ & 3,00 & 15,53 \\
Manuales cualificados y operarios de maquinaria & $4.756,22$ & 4,23 & 14,07 \\
Comerciantes y no cualificados servicios personales & $6.216,68$ & 5,32 & 16,34 \\
Auxiliares administrativos & $6.110,61$ & 7,62 & 15,85 \\
Profesionales cualificados & $8.806,08$ & 6,98 & 16,38 \\
Directivos & $12.172,57$ & 6,72 & 14,87 \\
Total & $5.804,03$ & 4,58 & 15,44 \\
\hline Fula
\end{tabular}

Fuente: elaboración propia a partir de la ENIGH 2016 (INEGI). 
de sus viviendas al trimestre no alcanza los $\$ 3.500$. En un segundo escalafón se agrupan los peones con los trabajadores manuales cualificados y operarios de maquinaria; el valor trimestral de sus viviendas se ubica en el espacio de los $\$ 4.000$. Un nivel superior lo ocupan los comerciantes y trabajadores no cualificados de servicios personales y los auxiliares administrativos, hogares cuyo valor trimestral de las rentas se sitúa alrededor de los $\$ 6.000$. En el extremo superior de la jerarquía ocupacional se encuentran los profesionales cualificados,que gastarían en renta al trimestre una media de $\$ 8.806,08$, y los directivos, que empelarían una media de $\$ 12.152,57$.

Para poder calibrar el peso que supone el gasto de la vivienda sobre el presupuesto familiar, se construyó el segundo indicador, la proporción del gasto en vivienda sobre los ingresos. Integra los gastos efectivos en renta de la vivienda y los gastos efectivos en cuotas mensuales de hipotecas de la vivienda que se está comprando, divididos por los ingresos corrientes. El análisis del estadístico de Levene mostró heterodasticidad de las varianzas, por lo que tuvo que aplicarse la prueba más robusta de Brown-Forsythe para confirmar la significatividad de las diferencias de medias. Atendiendo a los resultados, podemos apreciar el muy escaso peso que ocupa este gasto en los ingresos de los agricultores $(0,55 \%)$ y de los peones $(3 \%)$. Tenemos que recordar que una mayor proporción de ambos grupos de hogares por ocupaciones ya tenía su vivienda en propiedad y enteramente pagada, por lo que no incurrían en gastos en este tipo. Esta circunstancia baja la media de la proporción del gasto empeñado en vivienda sobre el total de ingresos. En el escalafón opuesto de la estructura ocupacional, los profesionales cualificados y los directivos empeñaban una proporción mayor de su presupuesto en los gastos en vivienda, el 6,98 y el 6,72 respectivamente.

Para corregir el anterior defecto, se procedió a eliminar de estos cálculos los hogares que ya eran propietarios efectivos de sus viviendas y no incurrían en gastos corrientes de este tipo. Así es como se pudo calcular el tercer indicador de la proporción corregida del gasto en vivienda sobre los ingresos, que integra solo los hogares con gastos por alquileres de la vivienda o con gastos por cuotas de hipotecas. El análisis de las varianzas ofreció un resultado similar al de los anteriores indicadores. Se encontró heterodasticidad, pero la prueba más robusta de Brown-Forsythe permitió establecer la significatividad de la diferencia de medias. Los resultados nos muestran que, excluidos los casos de los hogares propietarios de sus viviendas, las medias de las proporciones de gasto en vivienda se igualan mucho entre todas las clases sociales. Si acaso, se observa que los hogares encabezados por agricultores gastan de media en vivienda más que el resto de hogares, en concreto un $20,8 \%$ de su presupuesto.

Por último, se quiso comprobar la amplitud de los consumos en que incurren los hogares en el interior de las viviendas, y para ello se recurrió al gasto trimestral en energía.

El análisis mostró que las varianzas de las medias de cada uno de los grupos no eran homogéneas, por lo que se tuvo que recurrir al estadístico BrownForsythe para corroborar la significatividad estadística de las diferencias de medias. Los resultados muestran importantes diferencias en los consumos tri- 
Tabla 10. Diferencias de medias del gasto trimestral en energía según grupos de ocupaciones.

\begin{tabular}{lc}
\hline Ocupaciones & Gasto trimestral en energía \\
\hline Agricultores & 952,35 \\
Peones & 946,83 \\
Manuales cualificados y operarios de maquinaria & $1.188,35$ \\
Comerciantes y no cualificados servicios personales & $1.235,03$ \\
Auxiliares administrativos & $1.275,08$ \\
Profesionales cualificados & $1.455,29$ \\
Directivos & $1.963,09$ \\
Total & $1.206,35$ \\
\hline
\end{tabular}

Fuente: elaboración propia a partir de la ENIGH 2016 (INEGI).

mestrales en energía según las categorías ocupacionales. Aquí, los agricultores y los peones son quienes menos gastan en energía en sus viviendas, menos de $\$ 1.000$ al trimestre. Un segundo grupo de gasto integra los hogares de los trabajadores manuales cualificados, de los operarios de maquinaria, de los comerciantes, los trabajadores no cualificados de servicios personales y los auxiliares administrativos, que gastan alrededor de los \$1.200 trimestrales en energía. Ya entre las posiciones más altas, los profesionales cualificados gastan una media de $\$ 1.455,29$ en energía al trimestre y los directivos empeñan el doble que los grupos de ocupaciones más bajos en este gasto, una media al trimestre de $\$ 1.963,35$.

\section{Conclusiones}

Los resultados presentados delatan la particularidad del proceso de urbanización vivido por las urbes mexicanas en las tres últimas décadas, y que puede ser extensivo a buena parte de la región latinoamericana. La reciente incorporación de extensos territorios semirrurales a la urbe implica la inclusión en el contexto urbano de formas de habitabilidad y de relación con la vivienda propias del mundo rural. Estos espacios semirrurales permean la distribución clasista de la ciudad, ya que se aprecia que los escalafones más bajos de la estructura ocupacional encarnan esas formas de vida transicionales. Los agricultores y los peones viven de manera más señalada en localidades de menos de 2.500 habitantes, y lo hacen a través de fórmulas extrañas a los contextos urbanos altamente mercantilizados. Así, se recuerda que recurrían de forma preponderante a construir sus propias viviendas o a mandarlas construir (en lugar de recurrir al mercado inmobiliario), y que solían hacerlo con recursos propios en lugar de dirigirse a las instituciones financieras. Este rasgo incorpora una peculiaridad sobre la forma de crecimiento de las ciudades latinoamericanas. Al lado o al margen de los mecanismos institucionales y mercantiles propios de los contextos desarrollados de producción de espacio urbano, se evidencian fórmulas de ocupación de la tierra cercanas al contexto rural que tienen, por añadidura, una connotación clasista al ser propias de los escalafones más bajos de la estructura ocupacional. 
Esta peculiaridad matiza el sentido del propietario dentro de las grandes ciudades mexicanas, en contraste con lo consignado por la literatura. Si bien se señaló la importancia y las ventajas comparativas del acceso en régimen de propiedad de la vivienda, que solía ser coto exclusivo de las clases altas y medias, en los espacios semirrurales recién incorporados a las urbes mexicanas tenemos un buen número de propietarios que pertenecen a los escalones más bajos de la estructura ocupacional. Es cierto que este tipo de propiedades distan mucho de garantizar un pleno disfrute de las ventajas del territorio urbano, pues hablamos de viviendas con mayores niveles de hacinamiento, menos equipadas y en entornos alejados. Sin embargo, que un relevante número de agricultores y de peones disponga de sus propiedades evita tensiones añadidas sobre unos ingresos reducidos.

Por lo demás, se aprecia una ocupación y un disfrute del territorio urbano claramente escalonados según la estructura de ocupaciones. El consumo del espacio urbano se consigna también como un buen indicador de la jerarquía clasista en la ciudad. En la cúspide de esa jerarquía, los hogares encabezados por directivos son los que ocupan los espacios más aventajados de las ciudades bajo estudio. Estos hogares son mayoritariamente urbanos, disponen en gran parte de rentas suficientes para adquirir en el mercado sus viviendas sin tener que recurrir a hipotecas y adquieren casas con mejores condiciones de habitabilidad. Siempre a una distancia relevante, pero también en el escalafón más elevado, se sitúan los profesionales cualificados. Posteriormente, existe un grupo de hogares integrado por los auxiliares administrativos, los comerciantes y los trabajadores no cualificados de servicios personales y por los trabajadores cualificados que intercambian sus posiciones respecto a las diferentes condiciones de sus entornos residenciales, pero que se encuentran a una gran distancia respecto a las anteriores categorías ocupacionales. Todos estos hogares compondrían una precaria clase media urbana. En el extremo más bajo estarían los peones y los agricultores ya reseñados.

El presente trabajo sugiere la necesidad de atender a una de las modalidades que se están produciendo en la urbanización y que escapan a lo documentado por la literatura de los países desarrollados. La incorporación de espacios semirrurales a la urbe, que son el hábitat mayoritario para los hogares de agricultores y de peones, impone la necesidad de pensar en medidas adicionales para corregir los desequilibrios socioespaciales. Aparte de tenerse que enfrentar a las desigualdades derivadas de la extensión de los mercados inmobiliarios como agentes casi únicos de planificación urbana, las instituciones también deben generar políticas para mejorar las condiciones de esos espacios semirrurales, donde agricultores y peones recurren con sus escasos recursos para solventar con dificultades su acceso a la vivienda.

Sin duda, se requiere un mayor esfuerzo de investigación para determinar la formación clasista del espacio urbano desde el consumo de los entornos habitacionales. El presente estudio es un acercamiento preliminar a esta problemática. Se necesitan investigaciones de caso que confirmen, por un lado, las 
diferentes territorialidades urbanas propias de las distintas clases sociales; por otro, se requieren investigaciones cualitativas que muestren de manera detallada y en profundidad cómo ocurre el proceso clasista de identificación cognitiva y moral en un espacio de residencia.

\section{Referencias bibliográficas}

Aguilar, Adrián G.; Ward, Peter M. (2003). «Globalization, regional development, and mega-city expansion in Latin America: Analyzing Mexico City's peri-urban hinterland». Cities, 20 (1), 3-21. <https://doi.org/0.1016/S0264-2751(02)00092-6>.

Alıu, Ibrahim Rotimi; AJAla, Olayinka Akinsumbo (2015). «Residential polarization in a sub-Sahara African Megacity: an exploration study in Lagos». South African Geographical Journal, 97 (3), 264-286. $<$ https://doi.org/10.1080/03736245.2014.977810>.

Allen, Chris (2008). Housing Market Renewal and Social Class. Londres: Routledge.

Aristondo, Oihana; Onaindia, Eneritz (2018). "Inequality of energy poverty between groups in Spain». Energy, 153, 431-442. $<$ https://doi.org/10.1016/j.energy.2018.04.029>.

Avalle, Gerardo (2009). «Clases y territorio: construcción de subjetividades en los movimientos sociales». Ava. Revista de Antropología, 14, 1-19.

Bacqué, Marie-Hélène; Charmes, Éric; Vermeersch, Stéphanie (2014). «The Middle Class at Home among the Poor - How Social Mix is Lived in Parisian Suburbs: Between Local Attachment and Metropolitan Practices». International Journal of Urban and Regional Research, 38 (4), 1211-1233. $<$ https://doi.org/10.1111/1468-2427.12130>.

Ben-Shadar, Danny; Gabriel, Stuart; Golan, Roni (2018). «Housing affordability and inequality: A consumption-adjusted approach». Journal of Housing Economics (en prensa). $<$ https://doi.org/10.1016/j.jhe.2018.02.004>.

Benson, Michaela (2014). «Trajectories of middle-class belonging: The dynamics of place attachment and classed identities». Urban Studies, 51 (14), 3097-3112. $<$ https://doi.org/10.1177/0042098013516522>.

Blokland, Talja (2001). «Bricks, Mortar, Memories: Neighborhood and Networks in Collective Acts of Remembering». International Journal of Urban and Regional Research, 25 (2), 268-283. <https://doi.org/10.1111/1468-2427.00311>.

BRIDGE, Gary (2003). «Time-Space Trajectories in Provincial Gentrification». Urban Studies, 40 (12), 2545-2556. $<$ https://doi.org/10.1080/0042098032000136200>.

Bunting, Trudi; Walks, Alan R.; Filion, Pierre (2004). «The uneven geography of housing affordability stress in Canada metropolitan areas». Housing Studies, 19 (3), 361-393. <https://doi.org/10.1080/0267303042000204287>.

Burgess, Ernest W. (1925). «The Growth of The City: An Introduction to a Research Project». En: Park, Robert E.; Burgess, Ernest W.; McKenzie, Roderick D. (eds.). The City. Suggestions for Investigations of Human Behavior in the Urban Environment, 47-62. Chicago: University of Chicago Press.

Butler, Tim; Watt, Paul (2007). Understanding Social Inequality. Londres: Sage. 
CArdoso, María Mercedes (2018). «Territorios desiguales en el rururbano norte de la ciudad de Santa Fe. Análisis de la vulnerabilidad residencial y de la multiterritorialidad de productores de origen boliviano». Estudios Socioterritoriales. Revista de Geografía, 24, 1-18.

Castells, Manuel (1977). The Urban Question. A Marxist Approach. Londres: Edward Arnold Publishers.

Conley, Dalton (2001). «A Room with a View or a Room of One's Own? Housing and Social Stratification». Sociological Forum, 16 (2), 263-280.

DAvidson, Mark (2007). «Gentrification as global habitat: a process of class formation or corporate creation?». Transactions of the Institute of British Geographers, 32, 490-506. <https://doi.org/10.1111/j.1475-5661.2007.00269.x>.

Dowling, Robyn (2009). "Geographies of identity: landscapes of class». Progress in Human Geography, 33 (6), 833-839. <https://doi.org/10.1177/0309132508104998>.

Filandri, Marianna; Olagnero, Manuela (2014). «Housing Inequality and Social Class in Europe». Housing Studies, 29 (7), 977-993. $<$ https://doi.org/10.1080/02673037.2014.925096>.

Fleischer, Friederike (2007). "To Choose a House Means to Choose a Lifestyle. The Consumption of Housing and Class-Structuration in Urban China». City \& Society, 19 (2), 287-311. <https://doi.org/10.1525/city.2007.19.2.287>.

Gato, Maria Assunçao (2014). «Living in a(n) (un)Gated Community: Neighborhood Belonging in Lisbon's Parque das Naçoes». En: Watt, Paul; Smets, Peer (eds.). Mobilities and Neighborhood Belonging in Cities and Suburbs, 60-79. Nueva York: Palgrave MacMillan.

Gilbert, Alan; Ward, Peter M. (1985). Housing, the state and the urban poor: Policy and practice in three Latina American cities. Cambridge: Cambridge University Press.

Harvey, David (1985). The Urbanization of Capital: Studies in the History and Theory of Capitalist Urbanization. Baltimore: Johns Hopkins University Press.

Hunter, Albert (1987). "The Symbolic Ecology of Suburbia». En: Altman, Irwin; Wandersman, Abraham (eds.). Neighborhood and Community Environments, 191-222). Nueva York: Springer.

Instituto Nacional de Estadística y Geografía (2018). Delimitación de las zonas metropolitanas de México, 2015. Ciudad de México: INEGI.

Kelafas, Maria (2003). Working-Class Heroes. Protecting Home, Community and Nation in a Chicago Neighborhood. Los Angeles: University of California Press.

Lager, Debbie; Hoven, Bettina van; Huigen, Paulus P. P. (2013). «Dealing with change in old age: Negotiating working-class belonging in a neighborhood in a process of urban renewal in the Netherlands». Geoforum, 50, 54-61. <https://doi.org/10.1016/j.geoforum.2013.07.012>.

Lejeune, Zoe; Xhignesse, Guillaume; Krivoboков, Marko; Teller, Jacques (2016). "Housing quality as environmental inequality: the case of Wallonia, Belgium». Journal of Housing and the Built Environment, 31, 495-512. <https://doi.org/10.1007/s10901-015-9470-5>.

Lens, Michael C. (2018). "Extremely low-income households, housing affordability and the Great Recession». Urban Studies, 55 (8), 1615-1635. <https://doi.org/10.1177/0042098016686511>.

Libertun de Duren, Nora Ruth (2018). «Why there? Developers' rationale for building social housing in the urban periphery in Latin America». Cities, 72, 411-420. <https://doi.org/10.1016/j.cities.2017.10.006>. 
Matthews, Peter (2015). «Neighborhood Belonging, Social Class and Social Media -Providing Ladders to the Cloud». Housing Studies, 30 (1), 22-39. <https://doi.org/10.1080/02673037.2014.953448>.

McIntyre, Zhan; McKee, Kim (2012). «Creating sustainable communities through tenure-mix: the responsibilisation of marginal homeowners in Scotland». GeoJournal, 77, 235-247. <https://doi.org/10.1007/s10708-009-9322-5>.

McKeE, Kim; Muir, Jenny (2013). «An Introduction to the Special Issue - Housing in Hard Times: Marginality, Inequality and Class». Housing, Theory and Society, 30 (1), 1-9. <https://doi.org/10.1080/14036096.2012.682817>.

McKenzie, R. D. (1924). "The Ecological Approach to the Study of the Human Community». American Journal of Sociology, 30 (3), 287-301. $<$ https://doi.org/10.1086/213698>.

Mcloughlin, Fintan; Duffy, Aidan; Conlon, Michael (2012). «Characterizing domestic electricity consumption patterns by dwelling and occupant socio-economic variables: An Irish case study». Energy and Buildings, 48, 240-248. <https://doi.org/10.1016/j.enbuild.2012.01.037>.

Méndez-Lemus, Yadira; Vieyra, Antonio (2017). «How social capital enables or restricts the livelihoods of poor peri-urban farmers in Mexico». Development in Practice, 27 (3), 301-315. <https://doi.org/10.1080/09614524.2017.1296109>.

Meng, Gang; Hall, Brent G. (2006). "Assessing housing quality in metropolitan Lima, Peru». Journal of Housing and the Built Environment, 21, 413-439. <https://doi.org/10.1007/s10901-006-9058-1>.

Miller, Daniel; Jackson, Peter; Thrift, Nigel; Holbrook, Beverley; Rowlands, Michael (1998). Shoping, Place and Identity. Londres: Routledge.

Nates Cruz, Beatriz; Velásquez López, Paula Andrea (2019). «Gentrificación rururbana. Estudios territoriales en La Florida (Manizales-Villamaría) y Cerritos (Pereira), Colombia». Territorios, 41, 143-170. $<$ https://doi.org/10.12804/revistas.urosario.edu.co/territorios/a.6251>.

Oviedo Hernández, Daniel; Titheridge, Helena (2015). «Mobilities of the periphery: Informality, access and social exclusion in the urban fringe in Colombia». Journal of Transport Geography, 55, 152-164. <https://doi.org/10.1016/j.jtrangeo.2015.12.004>.

PARK, Robert E. (2015). «The City: Suggestions for the Investigation of Human Behavior in the City Environment». American Journal of Sociology, 20 (5), 577-612. <https://doi.org/10.1086/212433>.

Paton, Kirsteen (2014). Gentrification: A Working-Class Perspective. Farnham: Ashgate.

Pinkster, Feene, M. (2014). "I Just Live Here": Everyday Practices of Disaffiliation of Middle-class Households in Disadvantaged Neighborhoods». Urban Studies, 51 (4), 810-826. <https://doi.org/10.1177/0042098013489738>.

Pla, Jésica Lorena; Rodríguez de la Fuente, José Javier; Sacco, Nicolás (2018). «Clases sociales y condiciones de vida en el Gran Buenos Aires (2003-2013)». Revista Colombiana de Sociología, 41 (2), 189-218. <https://doi.org/10.15446/rcs.v41n2.64743>.

Pow, Choon-Piew (2009). «Neoliberalism and the Aestheticization of New Middle-Class Landscapes». Antipode, 41 (2), 371-390. <https://doi.org/10.1111/j.1467-8330.2009.00677.x>. 
Rhodes, James (2012). «Stigmatization, space, and boundaries in de-industrial Burnley». Ethnic and Racial Studies, 35 (4), 684-703. $<$ https://doi.org/10.1080/01419870.2011.591406>.

Rodenstedt, Ann (2014). Living in the calm and safe part of the city. The socio-spatial reproduction of upper-middle class neighborhoods in Malmö. Upsala: Uppsala Universitet.

Rojas-Caldelas, R.; Pena-Salmon, C.; Ranfla-González, A.; Venegas-Cardoso, R.; Leyva-Camacho, O.; Ley-García, J. (2010). "Approaches to analyzing the rural-urban interface: comprehensive development views from town and countryside». Transactions on Ecology and Environment, 129, 359-370. $<$ https://doi.org/10.2495/SC100 1>.

Savage, Mike (2010). «The Politics of Elective Belonging». Housing, Theory and Society, 27 (2), 115-135. <https://doi.org/10.1080/14036090903434975>.

- (2013). «Broken Communities?». En: Atkinson, Will; Roberts, Steven; SAvage, Mike (eds.). Class Inequality in Austerity Britain. Power, Difference and Suffering, 145-162. Londres: Palgrave MacMillan.

Selzer, Amy Kracker; Heller, Patrick (2010). «The spatial dynamics of middle-class formation in postapartheid South Africa: enclavization and fragmentation in Johannesburg». Political Power and Social Theory, 21, 171-208. <https://doi.org/10.1108/s0198-8719(2010)0000021012>.

ShefNer, Jon (2000). "Austerity and Neighborhood Politics in Guadalajara, Mexico». Sociological Inquiry, 70 (3), 338-359. <https://doi.org/10.1111/j.1475-682x.2000.tb00912.x>.

Solís, P.; BoAdo, M. (2016). Y sin embargo se mueve... Estratificación social y movilidad intergeneracional de clase en América Latina. Ciudad de México: El Colegio de México.

Sunega, Petr; Lux, Martin (2016). «Subjective perception versus objective indicators of overcrowding and housing affordability». Journal of Housing and the Built Environment, 31, 695-717. <https://doi.org/10.1007/s10901-016-9496-3>.

Urry, John (1995). «A middle-class countryside?». En: Butler, Tim; Savage, Mike (eds.). Social Change and the Middle Classes, 205-219. Londres: Routledge.

WARD, Peter (2004). "Informality of Housing Production at the Urban-Rural Interface: The 'Not so Strange Case' of the Texas Colonias». En: Roy, Ananya; AlSAYYAD, Nezar (eds.). Urban Informality: Transnational Perspectives from the Middle East, Latin America and South Asia, 243-270. Oxford: Lexington.

WatT, Paul (2006). «Respectability, Roughness and Race: Neighborhood Place Images and the Making of Working-Class Social Distinctions in London». International Journal of Urban and Regional Research, 30 (4), 776-797. <https://doi.org/10.1111/j.1468-2427.2006.00688.x>.

Wirth, Louis (1928). The Ghetto. Chicago: Chicago University Press.

Wood, Gavin; Ong, Rachel (2011). «Factors shaping the Dynamics of Housing Affordability in Australia 2001-06». Housing Studies, 26 (7-8), 1105-1127. <https://doi.org/10.1080/02673037.2011.615156>.

Zulaica, Laura; Oriolani, Federico (2018). "Quality of Life and Habitability Conditions in Peri-Urban Areas of Southern Mar de Plata, Argentina: A Multimethod Study». Applied Research in Quality of Life, 14, 659-683. <https://doi.org/10.1007/s11482-018-9618-z>. 